\title{
Clinical Impact of Flat Panel Volume CT Angiography in Evaluating the Accurate Intraoperative Deployment of Flow-Diverter Stents
}

\author{
(D) F. Clarençon, (D). Di Maria, (D). Gabrieli, (DE. Shotar, (D). Degos, (D)A. Nouet, (D)A. Biondi, and (D) N.-A. Sourour
}

\begin{abstract}
BACKGROUND AND PURPOSE: The deployment of flow-diverter stents may be difficult to analyse on regular DSA. The purpose of our study was to investigate the clinical impact of stent-dedicated flat panel volume CT angiography to evaluate intraoperatively the satisfactory deployment of flow-diverter stents.
\end{abstract}

MATERIALS AND METHODS: From January 2009 to April 2015, 83 consecutive patients (mean age, 51 years; 62 women) were treated in our institution with flow-diverter stents. Eighty-seven aneurysms (82 unruptured, 5 ruptured; 77 anterior, 10 posterior circulation) were treated in these 83 patients ( 4 patients had 2 aneurysms, both treated by means of flow-diverter stents). One patient was treated for a traumatic carotid cavernous fistula. In $80 \%$ of the cases (68/85) a flat panel volume CT angiography was performed in the angiographic suite just after the flow-diverter stent deployment. Stent visualization was assessed by 2 independent reviewers. The clinical impact of stent malapposition was evaluated.

RESULTS: Flow-diverter stent visualization was satisfactory in $73.5 \%$ of the cases. In 2 cases (2.9\%) the flat panel volume CT angiography prompted the operator to perform an additional intrastent angioplasty for a condition that was previously underestimated. Four patients (4.7\%) experienced acute thromboembolic complications; 3 others had delayed thromboembolic complications. Only 1 of these patients had thromboembolic complications (acute or delayed) related to stent misdeployment, which was easily managed intraoperatively with no clinical consequence.

CONCLUSIONS: Flat panel volume CT angiography is an interesting tool to depict flow-diverter stent misdeployment and may encourage the operator to perform intrastent angioplasty ( $2.9 \%$ of the cases in our experience) to reduce the risks of thromboembolic complications.

ABBREVIATIONS: FDS $=$ flow-diverter stent; $F P V-C T A=$ flat panel volume $C T$ angiography

$\mathbf{F}$ ow-diverter stents (FDSs) have gained acceptance in recent years for the treatment of intracranial aneurysms, especially giant and large ones. ${ }^{1,2}$ This new-generation stent shifted the paradigm of treatment for intracranial aneurysms and now helps in treating intracranial aneurysms that were previously considered as challenging or even impossible to treat by endovascular means. ${ }^{3}$ However, one of the drawbacks for their use is their poor radiopacity. ${ }^{4}$ Indeed, despite the addition of radiopaque strands in the design of these stents, their visibility remains poor, hampering the visualiza-

Received March 18, 2017; accepted after revision June 9.

From the Departments of Interventional Neuroradiology (F.C., J.G., E.S., N.-A.S.), Anesthesiology (V.D.), and Neurosurgery (A.N.), Pitié-Salpêtrière Hospital. Paris France; Paris VI University (F.C., J.G., E.S., V.D.), Pierre et Marie Curie, Paris. France; Department of Interventional Neuroradiology (F.D.M.), Foch Hospital, Suresnes, France; and Department of Neuroradiology and Endovascular Therapy (A.B.), Besançon University Hospital, Besançon, France.

Please address correspondence to Frédéric Clarençon, MD, PhD, Department of Neuroradiology, Pitié-Salpêtrière Hospital, 47 Bd de l'Hôpital, 75013 Paris, France; e-mail: fredclare5@msn.com

http://dx.doi.org/10.3174/ajnr.A5343 tion of their accurate deployment by the operator during the procedure. Flat panel volume CT angiography (FPV-CTA) is now increasingly used to evaluate the deployment of regular intracranial stents in cases of stent-assisted coiling, ${ }^{5-7}$ and has shown its effectiveness to depict kinking/misdeployment. However, the potential of this technique has been poorly reported for FDSs.

The purpose of our study was to evaluate the potential of the FPV-CTA to display the accurate deployment of FDSs and to evaluate the clinical impact of FDS misdeployment depicted by this technique.

\section{MATERIALS AND METHODS \\ Patient Demographics/Aneurysm Characteristics}

All patient demographics and aneurysm characteristics are summarized in Table 1. From January 2009 to April 2015, 87 intracranial aneurysms (82 unruptured, 5 acutely ruptured; 77 anterior, 10 posterior circulation) were treated in 83 consecutive patients (mean age, $51 \pm 12.1$ years; age range, $20-77$ years; 62 women, 21 men), during 85 embolization procedures ( 4 patients had 2 aneu- 
Table 1: Patient demographics and aneurysm characteristics

\begin{tabular}{|c|c|c|}
\hline Demographics/Characteristics & $\begin{array}{l}\text { Overall } \\
\text { Population }\end{array}$ & $\begin{array}{c}\text { Patients Who } \\
\text { Underwent FPV-CTA }\end{array}$ \\
\hline No. of patients & 83 & 68 \\
\hline Age (mean $\pm S D)$ & $51 \pm 12$ & $51 \pm 12$ \\
\hline Female (no., \%) & $62(75)$ & $51(75)$ \\
\hline No. of aneurysms & 87 & 70 \\
\hline Direct CC fistula (no.) & 1 & 1 \\
\hline No. of procedures & 84 & 70 \\
\hline \multicolumn{3}{|l|}{ Aneurysm locations } \\
\hline Anterior circulation (no., \%) & $76(87.4)$ & $63(90)$ \\
\hline Paraclinoid ICA (no., \%) & $36(41.4)$ & $28(40)$ \\
\hline Cavernous ICA (no., \%) & $20(23)$ & $17(24)$ \\
\hline ACA/AComA (no., \%) & $7(8)$ & $6(8.6)$ \\
\hline ICA terminus (no., \%) & $4(4.6)$ & $4(5.7)$ \\
\hline AChoA/PComA (no., \%) & $4(4.6)$ & $4(5.7)$ \\
\hline MCA (no., \%) & $4(4.6)$ & $3(4.3)$ \\
\hline Petrous ICA (no., \%) & $1(1.1)$ & $1(1.4)$ \\
\hline Posterior circulation & $11(12.6)$ & $7(10)$ \\
\hline Vertebral artery (no., \%) & $5(5.7)$ & $3(4.3)$ \\
\hline BA (no., \%) & $3(3.4)$ & $1(1.4)$ \\
\hline PCA (no., \%) & $2(2.3)$ & $2(2.9)$ \\
\hline SCA (no., \%) & $1(1.1)^{\prime}$ & $1(1.45)$ \\
\hline Aneurysm maximum diameter (mean $\pm \mathrm{SD}$ ) & $9.2 \pm 6.5$ & $9.8 \pm 6.5$ \\
\hline Aneurysm neck (mean $\pm S D$ ) & $5.4 \pm 3$ & $5.4 \pm 3$ \\
\hline Acutely ruptured aneurysms (no., \%) & $5(5.7)$ & $3(4.3)$ \\
\hline Recanalized aneurysms (no., \%) & $16(18.4)$ & $13(18.6)$ \\
\hline Previously clipped (no., \%) & $2(2.3)$ & $2(2.9)$ \\
\hline Previously coiled (no., \%) & $14(16.1)$ & $11(15.7)$ \\
\hline
\end{tabular}

Note:-ACA indicates anterior cerebral artery; AChoA, anterior choroidal artery; AComA, anterior communicating artery; BA, basilar artery; CC, carotid cavernous; PCA, posterior cerebral artery; PComA, posterior communicating artery; SCA, superior cerebellar artery.

rysms, both treated by means of FDS; in 2 cases, 2 aneurysms were treated with the same FDS). The mean maximum diameter of the aneurysms was $9.2 \pm 6.5 \mathrm{~mm}$; the mean neck size was $5.4 \pm 3 \mathrm{~mm}$. In 16 cases (18\%), FDS was used for the treatment of a recurrence in an aneurysm previously treated by coiling $(n=13 ; 14.9 \%)$, by FDS $(n=1 ; 1.1 \%)$, or clipping $(n=2 ; 2.3 \%)$. In addition, 1 patient (Patient \#43) was treated for a traumatic carotid cavernous fistula.

\section{Endovascular Treatment}

All procedures were performed under general anesthesia. In all except 1 case of blood-blister aneurysm, patients received dual antiplatelet therapy (clopidogrel and aspirin) before the treatment, which was pursued for 3-6 months after the procedure. Aspirin was then pursued for a total of 1 year. In all except 1 case (blood-blister aneurysm treated in emergency), the sensitivity to clopidogrel as well as to aspirin was evaluated by using the Multiplate aggregation test (Roche, Basel, Switzerland). In most cases, full anticoagulation was performed during the treatment, with an activated clotting time target between 2- to 3 -fold the baseline. For the patient treated in emergency for the blood-blister aneurysm, the antithrombotic protocol was as follows: oral clopidogrel, $300 \mathrm{mg}$, 4 hours before the endovascular treatment, then continuous IV heparin (activated clotting time, 2- to 3-fold the baseline), and finally an IV bolus of aspirin $(250 \mathrm{mg})$ during the procedure. Then the dual antiplatelet therapy (clopidogrel, $75 \mathrm{mg}$ and aspirin, $250 \mathrm{mg}$ ) was pursued for 6 months and aspirin alone for an 6 additional months.
For patients who were resistant to clopidogrel, clopidogrel was replaced by ticagrelor (90 mg, twice a day) for 6 months.

The patients were all treated with a FDS (Pipeline Embolization Device [Covidien, Irvine, California] in 58 cases [67\%], Silk [Balt Extrusion, Montmorency, France] in 14 cases [16\%], NeuroEndoGraft [Stryker Neurovascular, Kalamazoo, Michigan] in 10 cases [11.5\%], and FRED [MicroVention, Tustin, California] in 5 cases [5.7\%]). In 12/85 cases (14\%), 2 FDSs or more were used for the treatment of the same aneurysm. On average, $1.15 \pm 0.4$ FDSs were used per patient (range, 1-3).

In $80 \%$ of the cases (68/85), an FPVCTA was performed in the angiographic suite just after the stent(s) deployment. The remaining $20 \%$ of cases corresponded to the cases treated in our early experience for which no FPV-CTA was performed or to cases for which raw data were not available on the PACS. In 37\% of the cases (21/ 68 ), coils were already present in the aneurysm sac (either in case of recanalized coiled aneurysm or when the aneurysm was coiled before the FPV-CTA). In 15/68 cases $(22 \%)$, the delivery microcatheter was kept in place (ie, within the lumen of the FDS) during the FPVCTA acquisition (Fig 1). In one case, an FPV-CTA was performed with a jailed microcatheter inside the aneurysm sac (Fig 2).

All the patients explored by means of FPV-CTA had the same imaging protocol detailed below.

\section{Acquisition Protocol}

All FPV-CTAs were performed by using the same protocol. Via the guiding catheter used for the stent deployment, we proceeded to an intra-arterial injection in the parent vessel of iohexol, $300 \mathrm{mg} \mathrm{I} / \mathrm{mL}$, diluted at $20 \%$ with saline. All the FPVCTAs were performed in the same biplane angiographic suite (Axiom Artis dBC [Siemens, Erlangen, Germany]); C-arm FPV-CTA characteristics were as follow: $200^{\circ}$ of rotation (from $100^{\circ}$ left oblique projection to $100^{\circ}$ right oblique projection); 1 image $/ 0.4^{\circ}$ (20s-1KDR protocol). Twenty $\mathrm{mm}^{3}$ of the $20 \%$ diluted contrast media were injected at $1 \mathrm{~mL} / \mathrm{s}$ at the beginning of the CT-rotational acquisition. Secondary reconstructions were performed on a dedicated workstation (Artis Workplace; Siemens). For these reconstructions, a $512 \times 512$ matrix was used with a volume of view as small as possible, adapted to the length of the FDS. The voxel size was $0.14 \mathrm{~mm}$. A batch of about 500 images was obtained from this reconstruction, with 5-mm thick sections and no intersection gap.

\section{Evaluation Criteria}

All FPV-CTA acquisitions were independently reviewed by 2 observers (F.C. and F.D.M.) with 5 and 6 years, experience, respec- 

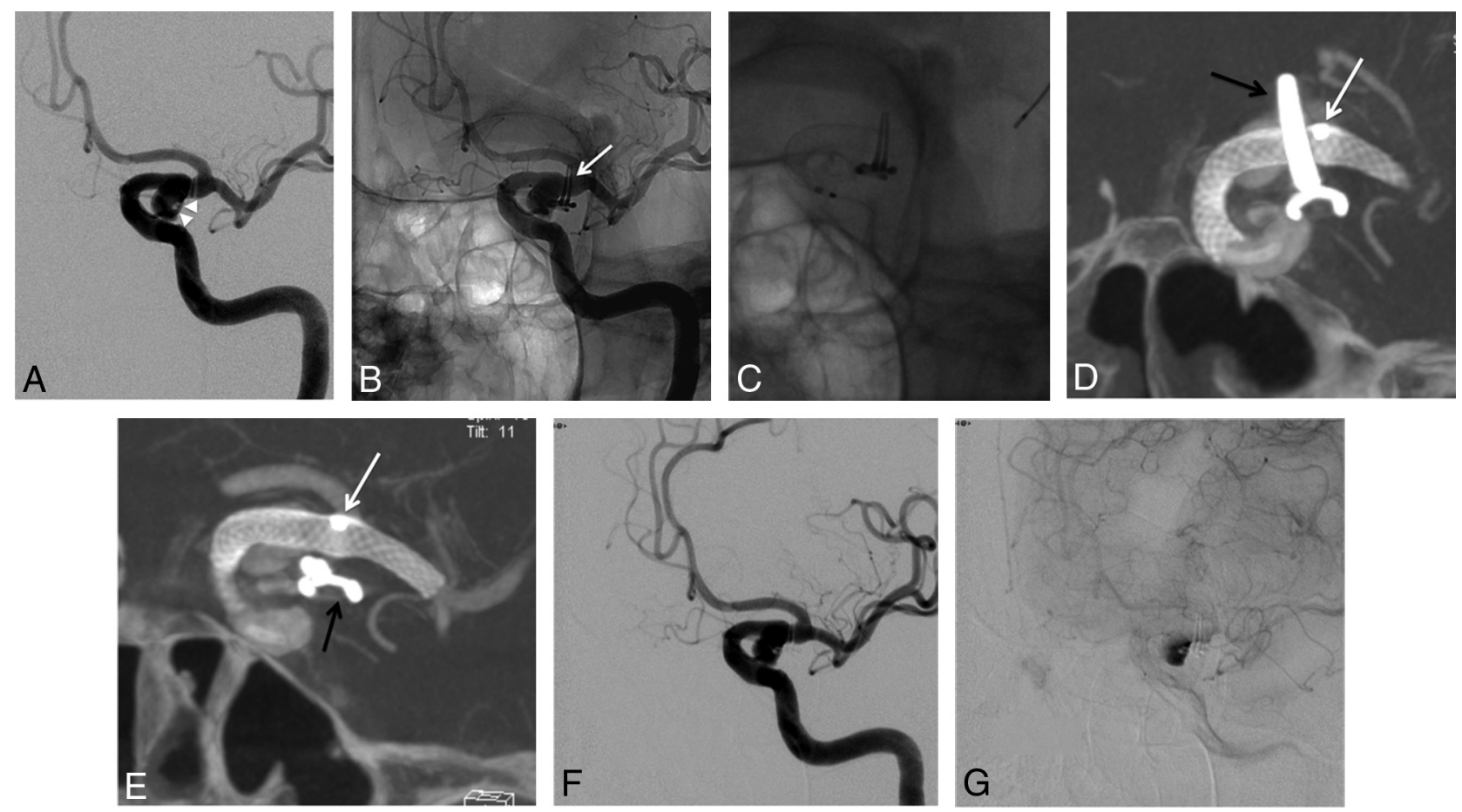

FIG 1. A 51-year-old woman presenting a recurrence of a left unruptured PComA aneurysm previously clipped. A, DSA in working projection showing the $5.2 \times 5.0 \mathrm{~mm}$ recurrence with a $4.7 \mathrm{~mm}$ neck (arrowheads). B, Unsubtracted snapshot of the DSA in working projection displaying the clip (white arrow). C, Unsubtracted snapshot during the deployment of the FDS (Pipeline Embolization Device) from the left M1 segment to the carotid siphon. $D$ and $E$, Snapshots from the FPV-CTA acquisition performed with $20 \%$ contrast media intra-arterial injection through the guiding catheter; MIP reconstruction. Satisfactory deployment of the stent is seen. Note the presence of the clip from the previous treatment (black arrows) and the microcatheter's tip, left in the FDS lumen during the acquisition (white arrows). Note that neither the clip nor the microcatheter's tip hamper the FDS visualization. $F$ and $G$, Final DSA in working projection after FDS deployment ( $F$, early phase; $G$, late phase). Stagnation of the contrast media within the aneurysm sac is seen at late phase $(G)$. PComA indicates posterior communicating artery.

tively, in interventional neuroradiology. The FPV-CTA volumes were analyzed on a dedicated workstation (Leonardo workstation) with the aid of thin section multiplanar reconstructions (mainly in a plane perpendicular to the great axis of the parent artery) and MIP (thickness, $5 \mathrm{~mm}$ ) reconstructions. The following criteria were independently evaluated by both observers: quality of the FDS visualization (poor/fair/good); the rate of the FDS visualized (not visualized/partial visualization/complete visualization); and the presence of misdeployment (kinking/misopening/twisted stent). In cases of stent misdeployment, the location of the misdeployment was graded as follows: proximal, central, or distal. Discrepancies between the 2 observers were settled in consensus. Finally, the presence of contrast media stagnation in the aneurysm sac was evaluated in consensus.

\section{Statistical Analysis}

Interrater agreement was performed by means of a weighted $\kappa$ test.

$\kappa$ index $\leq 0.40$ was considered as poor agreement; between 0.41-0.60 as moderate agreement; between $0.61-0.80$ as good agreement; and from $0.81-1$ as excellent agreement. ${ }^{8}$

Influence of the following factors on FDS visualization was evaluated: age, sex, aneurysm location (ie, anterior/posterior circulation), aneurysm maximum diameter, aneurysm neck, presence of coils/clips, microcatheter in place within the FDS lumen, and presence of contrast media stagnation in the aneurysm sac. All statistical tests were performed by means of the STATA soft- ware (Version 11; StataCorp, College Station, Texas). A $P$ value $<.05$ was considered as statistically significant.

\section{Ethical Statement}

Neither approval of the institutional review board nor patient informed consent are required by the ethics committee of our institution (Pitié-Salpêtrière Hospital) for retrospective analyses of patient records and imaging data.

\section{RESULTS \\ FDS Visualization}

Data regarding FDS evaluation are summarized in Table 2.

Interrater agreement was fair $(k=0.38)$ for the stent visualization quality (poor/fair/good) on FPV-CTA, moderate $(k=$ $0.49)$ for the proportion of the stent visualized (partial/complete), and moderate $(k=0.57)$ for the analysis of the deployment quality (satisfactory/misopening). The analysis in consensus showed a satisfactory visualization of the FDS in $83.8 \%$ of the cases, a complete visualization of the FDS in $73.5 \%$ of the cases, and a misopening of the FDS in $11.8 \%$ of the cases (Figs 3 and 4 ). Univariate analysis showed that only the aneurysm's maximum diameter had an influence on stent visualization $(P<.001)$. On the contrary, age, sex, aneurysm location (ie, anterior/posterior circulation), presence of coils/clipping, stent type, and number of stents had no influence on the visualization quality of the FDS (Table 3). In addition, the presence of the microcatheter inside the FDS during the acquisition (observed in 15/68 cases [22\%]), the 

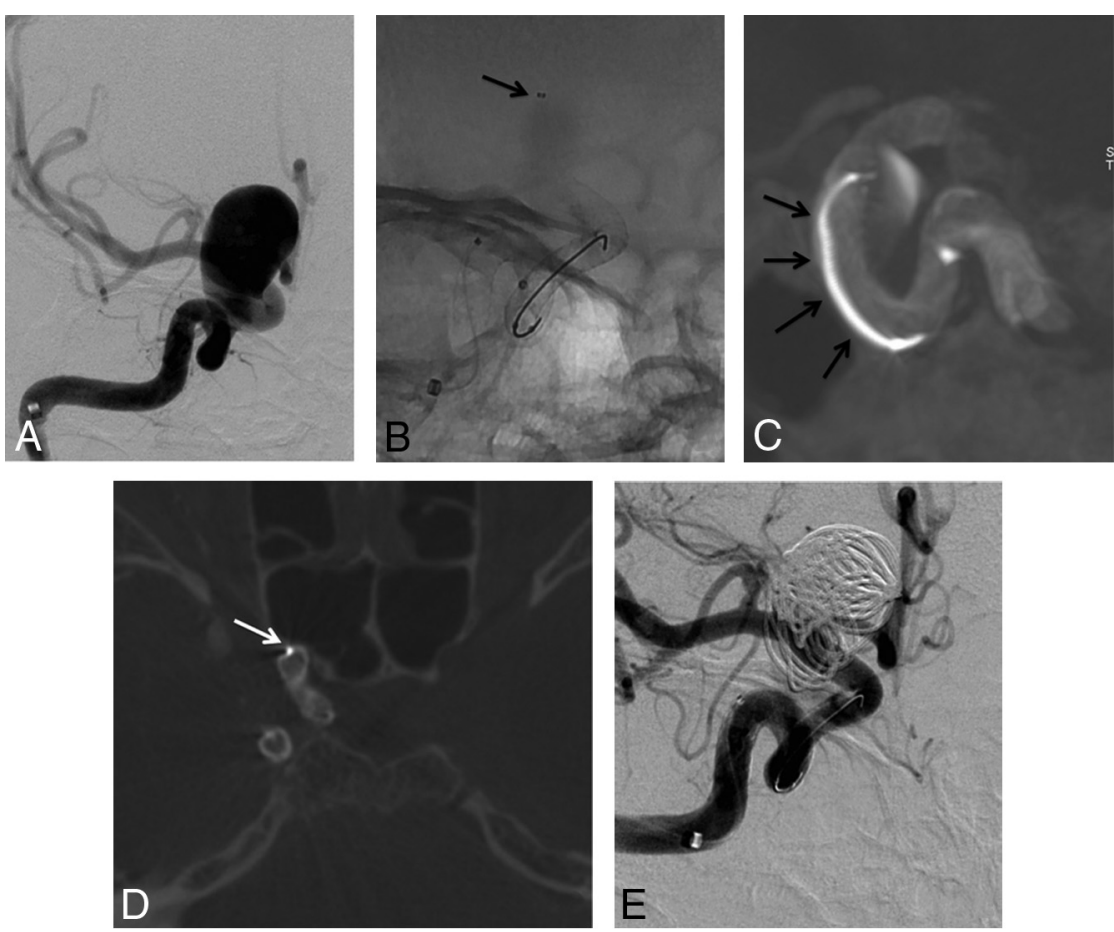

FIG 2. A 40-year-old woman treated for an incidental large right carotid ophthalmic aneurysm. $A$, Right ICA DSA in working projection showing the large paraclinoid aneurysm. B, Plain x-ray snapshot in working projection after the deployment of the FDS (Pipeline Embolization Device) in the parent artery, with a microcatheter jailed in the aneurysm sac (arrow). C, FPV-CTA acquisition, MIP reconstruction in sagittal view showing the satisfactory deployment of the FDS with the microcatheter jailed between the FDS and the wall of the parent artery (arrows). D, FPV-CTA, axial view. The jailed microcatheter is seen between the FDS and the wall of the parent artery (arrow). $E$, Final control DSA in working projection after loose coiling of the sac. Satisfactory exclusion of the sac is seen.

\section{Table 2: Interrater agreements and analysis in consensus for the} evaluation of FPV-CTAs

\begin{tabular}{lr}
\hline Interrater agreements & \\
Stent visualization quality & $\kappa=0.38$ \\
Stent rate visualized & $\kappa=0.49$ \\
Misdeployment & $\kappa=0.57$ \\
Analysis in consensus & \\
Good visualization & $83.8 \%$ \\
Fair/poor visualization & $16.2 \%$ \\
Complete visualization & $73.5 \%$ \\
Partial visualization & $27 \%$ \\
Satisfactory opening & $88.2 \%$ \\
Misdeployment & $11.8 \%$ \\
\hline
\end{tabular}

presence of a jailed microcatheter (observed in $1 / 68$ cases [ $1.5 \%]$ ), or the presence of contrast media stagnation (observed in 4/68 cases $[6 \%]$ ) had no influence on visualization quality of the FDS (Table 3). Multivariate analysis showed that both the presence of coils inside the aneurysm sac and an intrasaccular contrast media stagnation during the FPV-CTA decreased the visualization quality of the FDS ( $P=.015$ and .02 , respectively).

\section{FDS Misopening/Additional Angioplasty}

Misopening was depicted on FPV-CTA in $11.8 \%(8 / 68)$ of the cases. In $2.9 \%(2 / 68)$ of the cases, the misopening was visible only on the FPV-CTA. On the contrary, no case of malapposition was seen only on plain $x$-ray and missed by the FPV-CTA. No malapposition was seen in the case with a jailed microcatheter. In 2/8 cases (25\%), the misopening was located at the distal aspect of the FDS; in $3 / 8$ cases $(37.5 \%)$, it was located at the proximal aspect. In the remaining 3 cases (37.5\%) the misopening was observed in the central aspect of the FDS.

\section{Clinical Consequences of Misdeployment Depicted}

Seven (10.3\%) intrastent angioplasties were performed after FDS deployment. In 5 cases $(7.4 \%)$, the angioplasty was performed because major misopening was depicted on DSA before the FPVCTA was performed. In the remaining 2 cases $(2.9 \%)$, the misopening that led the operator to perform angioplasty was only seen on FPV-CTA (Figs 3 and 4). Interestingly, in both cases, the FDS were NeuroEndoGrafts.

\section{Complications}

Overall thromboembolic complication rate was $9.4 \%(8 / 85)$; the symptomatic thromboembolic complication rate was $7 \%(6 / 85)$. Thromboembolic complications leading to neurologic sequelae were recorded in $4.7 \%(4 / 85)$ of the cases. Acute thromboembolic complications (ie, intraoperative or in the immediate postoperative period) occurred in $4.7 \%$ of the cases (4/85); among them, only $2(2.3 \%)$ were responsible for neurologic sequelae. Only 1 (1.2\%) thromboembolic complication related to misopening or kinking of the FDS was recorded. In this case, a clot formed inside the FDS, which was misopened in its central aspect. This incomplete opening was obvious on DSA, and the clot resolved after intrastent angioplasty and IV injection of a bolus of abciximab. No clinical consequence was recorded in the latter patient. Three delayed ( $\geq 4$ months) ischemic complications were recorded. These 3 cases have been previously reported. ${ }^{9}$ None of these delayed ischemic complications were related to FDS misopening or kinking. Interestingly, in 1 case, a delayed stenosis located at the distal aspect of the FDS was depicted after a thromboembolic event occurred, whereas no misopening was seen on the FPV-CTA performed intraoperatively. ${ }^{9}$

\section{DISCUSSION}

FDSs are a new tool in the armamentarium of the interventional neuroradiologist that may help in treating intracranial aneurysms previously considered as uncoilable or very challenging to treat with regular techniques. ${ }^{10}$

Despite their potential for the treatment of difficult aneurysms, one of the major limitations for their use is their poor radiopacity. Indeed, to increase their pushability and conformability, the use of radiopaque components in the design of the FDS is limited because they dramatically increase their stiffness. ${ }^{11}$ 

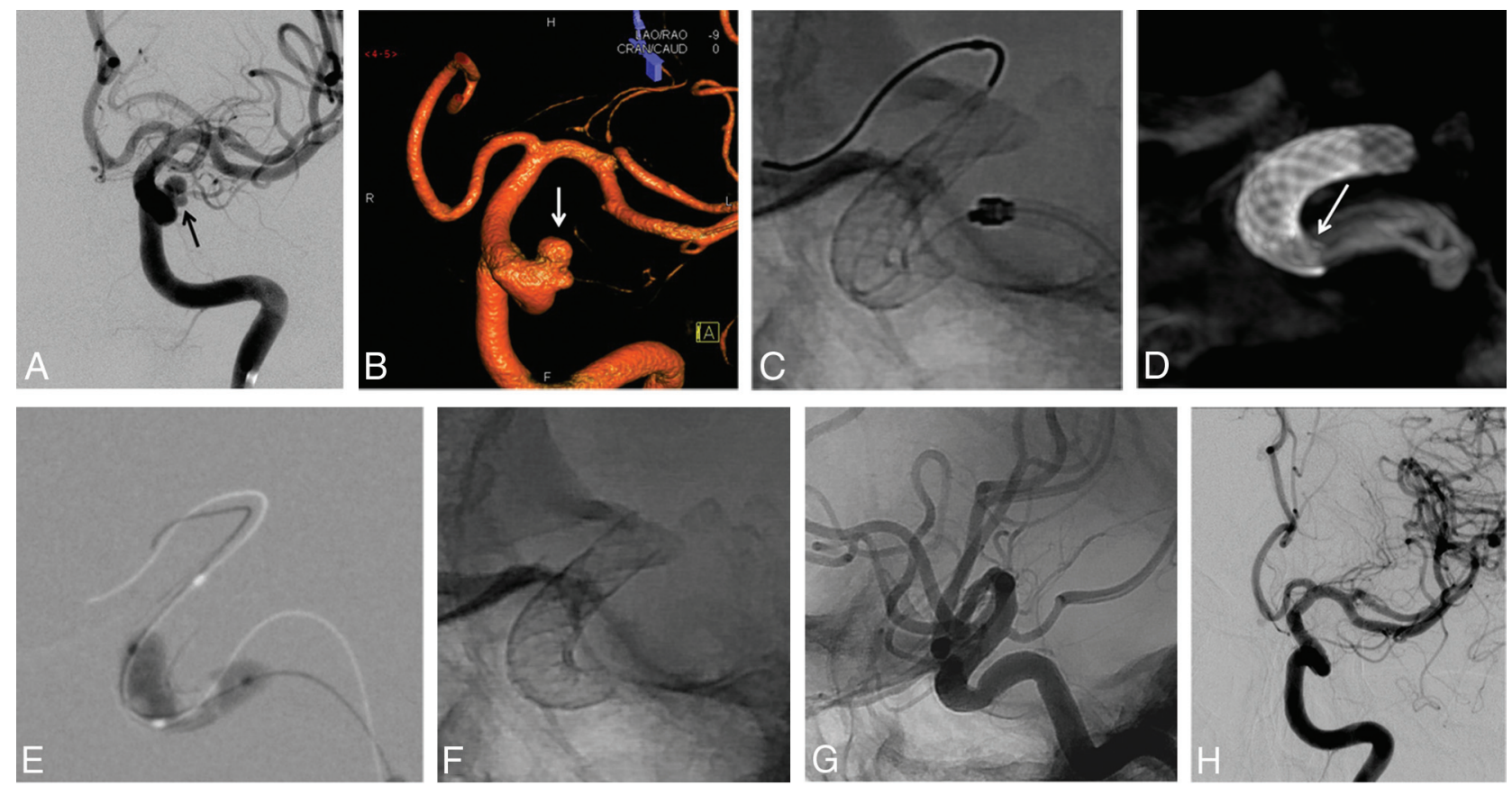

FIG 3. A 52-year-old woman treated for an unruptured left carotid ophthalmic large-neck aneurysm. A, Left ICA DSA in working projection (A) and $3 \mathrm{D}$ rotational angiography $(B)$ showing the bi-lobed paraclinoid aneurysm with a large neck. Two overlapped FDSs (NeuroEndoGraft) were deployed in the carotid siphon to cover the aneurysm neck. C, Unsubtracted plain x-ray in lateral projection; the satisfactory opening of the distal FDS is demonstrated, but the proximal aspect of the proximal FDS is not clearly seen. D, FPV-CTA (sagittal view MIP reconstruction clearly separates the 2 FDSs and confirms an incomplete opening of the proximal aspect of the proximal FDS (arrow), which was subsequently treated by intrastent balloon angioplasty $(E)$. $F$ and $G$, Plain $x$-ray snapshots ( $F$, without and $G$, with contrast media injection) in lateral projection showing a satisfactory opening of the FDS. H, One-year follow-up DSA in working projection showing the complete occlusion of the aneurysm.
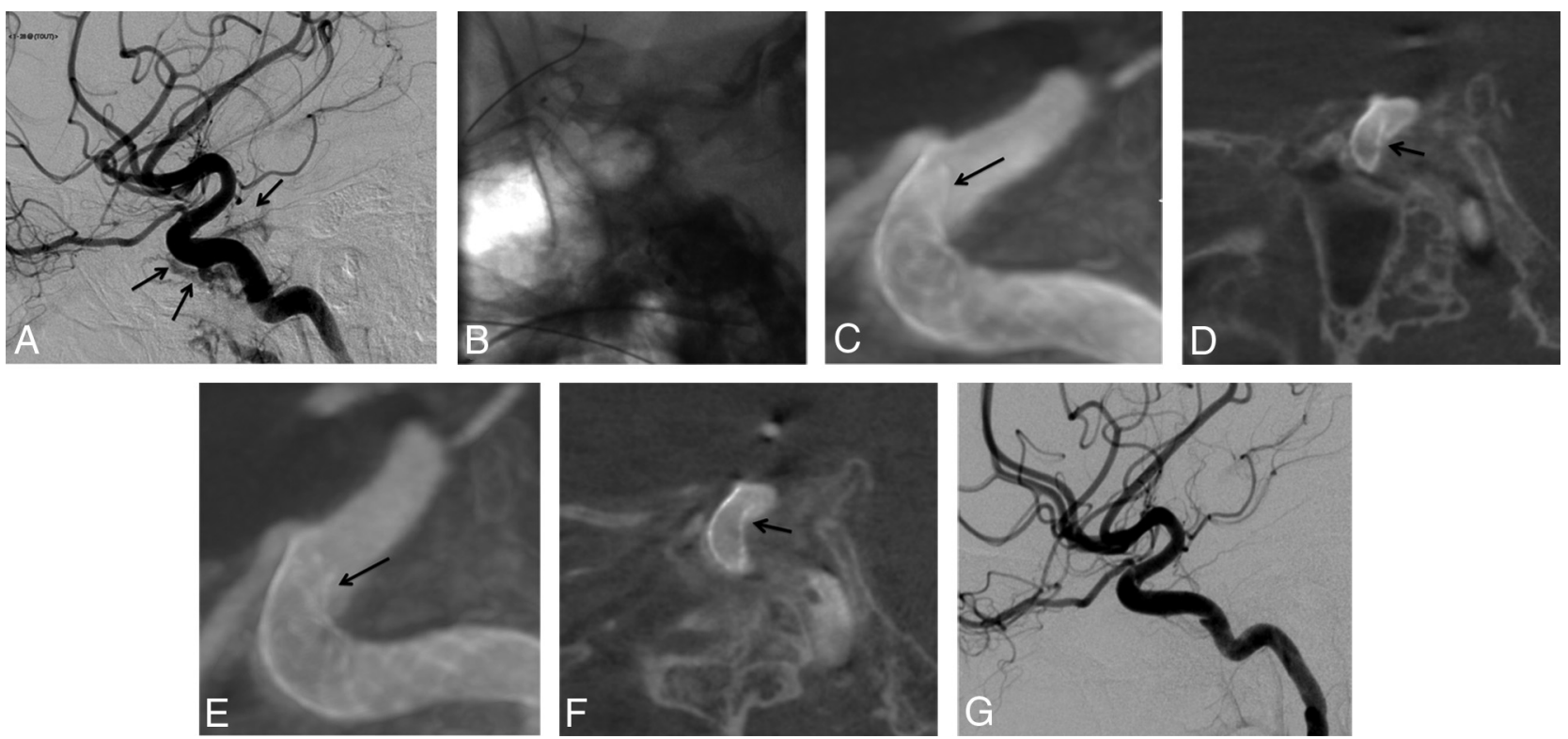

FIG 4. A 30-year-old man treated for a traumatic left carotid cavernous fistula, 1 month after a severe traumatic brain injury. A, Left ICA DSA in lateral projection before stent placement. Note the filling of the ipsilateral cavernous sinus (arrows). Treatment with an FDS covering the ICA's arterial tear was chosen. One FDS (NeuroEndoGraft) was deployed in the carotid siphon to cover the arterial tear. B, Unsubtracted snapshot after the stent deployment. No obvious malapposition of the stent is seen. $C$ and D, FPV-CTA after the stent deployment in sagittal MIP reconstruction $(C)$ and thin section sagittal oblique reconstruction $(D)$. This acquisition clearly shows a narrowing of the distal aspect of the FDS (arrow) that prompted the operator to perform an intrastent balloon angioplasty. $E$ and F, Postangioplasty FPV-CTA (E, sagittal view, MIP reconstruction; $F$, thin-section sagittal oblique reconstruction) shows incomplete but satisfactory opening of the distal aspect of the FDS (arrow). G, One-year follow-up DSA showing the complete cure of the traumatic fistula.

FPV-CTA is a CT-like acquisition performed via a rotation of the C-arm-mounted flat panel around the patient's head that can be easily performed in the angiographic suite. Since its early de- velopment, it has shown its potential to depict intraprocedural bleeding $^{12}$ and also to provide accurate imaging of endovascular implants. ${ }^{13,14}$ The potential of FPV-CTA has been shown for the 


\begin{tabular}{|c|c|c|c|c|}
\hline Variables & $\begin{array}{l}\text { Poor/Fair FDS } \\
\text { Visualization }\end{array}$ & $\begin{array}{c}\text { Good FDS } \\
\text { Visualization }\end{array}$ & $\begin{array}{c}\text { Univariate } \\
\text { Analysis } \\
\text { (P Value) }\end{array}$ & $\begin{array}{c}\text { Multivariate } \\
\text { Analysis } \\
\text { (P Value) }\end{array}$ \\
\hline Age, yr (mean $\pm S D)$ & $51.5 \pm 12.7$ & $51.5 \pm 12.3$ & .12 & .7 \\
\hline Female (no., \%) & $7(64)$ & $44(77)$ & .45 & .61 \\
\hline Anterior location (no., \%) & $10(90)$ & $51(89)$ & 1 & .94 \\
\hline Acute rupture (no., \%) & $0(0)$ & $3(5)$ & 1 & .64 \\
\hline Recanalization/recurrence & $4(36)$ & $7(12)$ & .73 & .34 \\
\hline Aneurysm max. diameter, $\mathrm{mm}($ mean $\pm \mathrm{SD})$ & $15.5 \pm 8.2$ & $8.7 \pm 5.2$ & $<.001^{\mathrm{a}}$ & .06 \\
\hline Aneurysm neck, mm (mean $\pm \mathrm{SD}$ ) & $6.35 \pm 3.65$ & $5.1 \pm 2.2$ & .13 & .795 \\
\hline Stent type (PED; no., \%) & $36(63)$ & $8(73)$ & .73 & .96 \\
\hline No. of stents (mean $\pm \mathrm{SD}$ ) & $1.2 \pm 0.5$ & $1.1 \pm 0.3$ & .25 & .92 \\
\hline Additional coils (no., \%) & $8(73)$ & $14(25)$ & $.004^{\mathrm{a}}$ & $.015^{\mathrm{a}}$ \\
\hline Microcatheter in place (no., \%) & $2(18)$ & $11(19)$ & 1 & .28 \\
\hline Contrast media stagnation (no., \%) & $2(18)$ & $2(3.5)$ & .12 & $.02^{\mathrm{a}}$ \\
\hline
\end{tabular}

Note:-max indicates maximum; PED, Pipeline Embolization Device.

${ }^{\text {a }}$ Statistically significant difference.

accurate visualization of intracranial stents (laser-cut ${ }^{5,15,16}$ as well as braided stents ${ }^{7}$ ). It helps to see precisely the struts of the stent and may allow the depiction of a kinking, twisting, or stent misopening. FPV-CTA protocols have been developed either without injection $^{5}$ or with diluted contrast material injection. ${ }^{17,18}$ The major advantage of protocols with contrast material injection (IV or intra-arterial) is that they may help to depict intrastent thrombus or endoleak (ie, contrast media filing between the FDS and the inner wall of the parent artery) caused by incorrect apposition of the stent.

Only scant data are available in the literature on the potential of FPV-CTA by intra-arterial ${ }^{4,7}$ or IV $^{17}$ injection for the visualization of FDSs and its clinical impact. Such protocols have been used to evaluate the accuracy of the FDS deployment intraoperatively ${ }^{4}$ and for follow-up imaging. ${ }^{4,17}$ The potential of the visualization of the accurate deployment of FDS appears of tremendous importance owing to the fact that most intraoperative, postprocedure, or delayed complications, like thromboembolic events or even stent occlusion, seem to be related to FDS kinking or misdeployment. ${ }^{19}$ In our series, we did not record any symptomatic ischemic complication caused by kinking or misdeployment of the FDS. The absence of such complications in our series may be explained by the fact that we widely used FPV-CTA ( $80 \%$ of the cases) to evaluate the FDS deployment and performed intrastent angioplasty in case of inaccurate wall apposition of the FDS. In addition, some cases of delayed aneurysm rupture have been linked to endoleak caused by incomplete deployment of the FDS. These endoleaks may lead to filling of the aneurysm with no exit for the blood trapped in the aneurysm sac. ${ }^{20}$ No such complication occurred in our series. Consequently, we think that FPVCTA should be systematically performed after FDS deployment because it may reduce the complication rate observed with these devices, one of the main drawbacks for their use.

To the best of our knowledge, we present the largest series of patients treated by FDS who underwent intraoperative FPV-CTA, and the only one for which an interrater agreement was evaluated and for which risk factors for poor/fair FDS visualization were assessed. This protocol provides, in a single acquisition, information on stent deployment, intrastent thrombosis, or stenosis and helps to see precisely which side arteries are covered by the FDS. We chose intra-arterial rather than an IV contrast media injection for the FPV-CTA acquisition to reduce the risk of poor opacification of the parent artery because of nonproper acquisition time. In addition, a $20 \%$ contrast material dilution $(20 \%$ contrast me$\mathrm{dia} / 80 \%$ saline) was chosen to avoid bright opacification of the parent vessel that would have hampered the visualization of the FDS.

In our series, satisfactory visualization of the FDS was reached in $83.8 \%$ of the cases; complete FDS visualization was observed in $73.5 \%$ of the cases. Interrater agreement was poor for stent visualization quality $(k=0.38)$ and moderate for the rate of FDS visualization $(k=0.49)$. However, the depiction of misopening was more reproducible $(k=0.57)$. Malapposition of the FDS was observed in $11.8 \%$ of the cases, which prompted the operator to perform an intrastent angioplasty in $7.4 \%$ of the cases $(2.9 \%$ on the basis of FPV-CTA alone). In the series by Kizilkilic et $\mathrm{al}^{4}{ }^{4}$ FPV-CTA results led to additional maneuvers (intrastent angioplasty or telescopic stent deployment) in $13 \%$ of the cases.

The only factors that were found by the multivariate analysis to be reducing the FDS visualization were the presence of coils and the presence of contrast media stagnation $(P=.015$ and .02 , respectively). Consequently, we recommend performing the FPVCTA acquisition before additional coiling when coils are needed (especially for large and giant aneurysms). To avoid contrast media stagnation in the aneurysm sac (frequently seen in giant and large aneurysms) that may hamper the FDS visualization, we recommend rinsing the parent artery with saline through the guiding catheter before performing the FPV-CTA. However, this rinsing may sometimes be insufficient when major intrasaccular stagnation is obtained after the FDS deployment. Interestingly, FPVCTA acquisitions with the microcatheter inside the FDS lumen did not reduce the visualization quality. In addition, in the only case in which a jailed microcatheter was present in our series, no malapposition was observed. However, the presence of a jailed microcatheter may hamper the FDS deployment, especially in cases of the parent artery presenting huge curves.

\section{Limitations of the Study}

Our study presents some limitations. First, this is a monocenter retrospective study. Second, the population involved was a middle-sized one $(n=83)$. However, the relatively low volume of the population involved could be explained by the fact that the indi- 
cations for FDS treatment are still nowadays limited because of a significant increased complication risk compared with regular endovascular techniques. ${ }^{21}$ Second, no metallic artifact reduction algorithm was used in our series. These algorithms may be very useful when coils or clipping are already present before the stent deployment (recanalized aneurysms). ${ }^{6,14,22}$ However, our results reflect the regular clinical practice because these algorithms are only available in few centers with angiographic suites optimized to clinical research. Finally, the proportion of FDS brands used for the treatment of the patients was not evenly distributed. Indeed, in most cases, a Pipeline Embolization Device was used (58/85; $68 \%$ ). This heterogeneity made the comparison between the different FDSs for the quality of the deployment impossible.

\section{CONCLUSIONS}

FPV-CTA is a useful tool to depict FDS misdeployment/misopening, which may be not visible on plain x-ray. In $2.9 \%$ of the cases, the results of the FPV-CTA prompted investigators to perform intrastent angioplasty to avoid acute or delayed thromboembolic complication. We thus recommend performing FPV-CTA systematically after stent deployment in patients treated with FDS.

Disclosures: Frédéric Clarençon-UNRELATED: Payment for Lectures including Service on Speakers Bureaus: Medtronic, Guerbet, Balt Extrusion; Other: Codman Neurovascular, Comments: Study Core Lab. Alessandra Biondi-RELATED: Consulting Fee or Honorarium: Medtronic, Stryker Neurovasular, Comments: consultant and proctor; UNRELATED: Consultancy: Phenox. Nader-Antoine Sourour-RELATED: Consulting Fee or Honorarium: Medtronic, Balt Extrusion, Microvention; UNRELATED: Stock/Stock Options: Medina.

\section{REFERENCES}

1. Briganti F, Napoli M, Tortora F, et al. Italian multicenter experience with flow-diverter devices for intracranial unruptured aneurysm treatment with periprocedural complications-a retrospective data analysis. Neuroradiology 2012;54:1145-52 CrossRef Medline

2. Brinjikji W, Murad MH, Lanzino G, et al. Endovascular treatment of intracranial aneurysms with flow diverters: a meta-analysis. Stroke 2013;44:442-47 CrossRef Medline

3. Brinjikji W, Cloft HJ, Fiorella D, et al. Estimating the proportion of intracranial aneurysms likely to be amenable to treatment with the Pipeline embolization device. J Neurointerv Surg 2013;5:45-48 CrossRef Medline

4. Kizilkilic O, Kocer N, Metaxas GE, et al. Utility of VasoCT in the treatment of intracranial aneurysm with flow-diverter stents. J Neurosurg 2012;117:45-49 CrossRef Medline

5. Clarençon F, Piotin M, Pistocchi S, et al. Evaluation of stent visibility by flat panel detector CT in patients treated for intracranial aneurysms. Neuroradiology 2012;54:1121-25 CrossRef Medline

6. van der Bom IM, Hou SY, Puri AS, et al. Reduction of coil mass artifacts in high-resolution flat detector conebeam CT of cerebral stent-assisted coiling. AJNR Am J Neuroradiol 2013;34:2163-70 CrossRef Medline
7. Poncyljusz W, Zwarzany E, Safranow K. Visualization of novel microstents in patients with unruptured intracranial aneurysms with contrast-enhanced flat panel detector CT. Eur J Radiol 2015;84: 1313-17 CrossRef Medline

8. Landis JR, Koch GG. The measurement of observer agreement for categorical data. Biometrics 1977;33:159-74 CrossRef Medline

9. Guédon A, Clarençon F, Di Maria F, et al. Very late ischemic complications in flow-diverter stents: a retrospective analysis of a single-center series. J Neurosurg 2016;125:929-35 CrossRef Medline

10. Wakhloo AK, Gounis MJ. Revolution in aneurysm treatment: flow diversion to cure aneurysms: a paradigm shift. Neurosurgery 2014;61 Suppl 1:111-20 CrossRef Medline

11. Hoffmann T, Gugel S, Beuing O, et al. Radiopacity assessment of neurovascular implants. Current Directions in Biomedical Engineering 2016;2:533-36 CrossRef

12. Struffert T, Richter G, Engelhorn T, et al. Visualisation of intracerebral haemorrhage with flat-detector CT compared to multislice CT: results in 44 cases. Eur Radiol 2009;19:619-25 CrossRef Medline

13. Psychogios MN, Scholz B, Rohkohl C, et al. Impact of a new metal artefact reduction algorithm in the noninvasive follow-up of intracranial clips, coils, and stents with flat-panel angiographic CTA: initial results. Neuroradiology 2013;55:813-18 CrossRef Medline

14. Stidd DA, Theessen H, Deng Y, et al. Evaluation of a metal artifacts reduction algorithm applied to postinterventional flat panel detector CT imaging. AJNR Am J Neuroradiol 2014;35:2164-69 CrossRef Medline

15. Levitt MR, Cooke DL, Ghodke BV, et al. "Stent view" flat-detector CT and stent-assisted treatment strategies for complex intracranial aneurysms. World Neurosurg 2011;75:275-78 CrossRef Medline

16. Richter G, Engelhorn T, Struffert T, et al. Flat panel detector angiographic CT for stent-assisted coil embolization of broad-based cerebral aneurysms. AJNR Am J Neuroradiol 2007;28:1902-08 CrossRef Medline

17. Yu SC, Lee KT, Lau TW, et al. Intravenous C-arm conebeam CT angiography following long-term flow-diverter implantation: technologic evaluation and preliminary results. AJNR Am J Neuroradiol 2016;37:481-86 CrossRef Medline

18. Ebrahimi N, Claus B, Lee CY, et al. Stent conformity in curved vascular models with simulated aneurysm necks using flat-panel CT: an in vitro study. AJNR Am J Neuroradiol 2007;28:823-29 Medline

19. Benndorf G, Strother CM, Claus B, et al. Angiographic CT in cerebrovascular stenting. AJNR Am J Neuroradiol 2005;26:1813-18 Medline

20. Darsaut TE, Rayner-Hartley E, Makoyeva A, et al. Aneurysm rupture after endovascular flow diversion: the possible role of persistent flows through the transition zone associated with device deformation. Interv Neuroradiol 2013;19:180-85 CrossRef Medline

21. Naggara ON, Lecler A, Oppenheim C, et al. Endovascular treatment of intracranial unruptured aneurysms: a systematic review of the literature on safety with emphasis on subgroup analyses. Radiology 2012;263:828-35 CrossRef Medline

22. Chintalapani G, Chinnadurai P, Srinivasan V, et al. Evaluation of $\mathrm{C}$-arm CT metal artifact reduction algorithm during intra-aneurysmal coil embolization: assessment of brain parenchyma, stents and flow-diverters. Eur J Radiol 2016;85:1312-21 CrossRef Medline 